\title{
An Improved EEG De-noising Approach in Electroencephalogram (EEG) for Home Care
}

\author{
Hong Peng, Bin Hu, Yanbing Qi, Qinglin Zhao \\ The School of Information Science and Engineering \\ Lanzhou University \\ Lanzhou, China
}

\{bh, pengh\}@1zu.edu.cn; qiyb09@1zu.edu.cn

\author{
Martyn Ratcliffe \\ The School of Computing, Telecommunications and \\ Networks \\ Birmingham City University \\ Birmingham, UK \\ Martyn.Ratcliffe@bcu.ac.uk
}

\begin{abstract}
Mental health care is becoming an increasing concern in home care projects. As an integral part of Telecare and Telehealth systems, portable EEG recording and real-time analysis are increasingly being used as non-intrusive monitoring techniques. In home environments without the supervision of a physician and absence of electromagnetic shielding, the raw EEG data, especially the most important alpha rhythm, which can be used to detect the mental illness and depression, is polluted by background noise such as Ocular Artifacts (OA), DC adrift and so on. In this paper, the raw data is processed in two steps: step one is a pre-process to remove $\mathrm{DC}$ adrift and $50 / 60 \mathrm{~Hz} \mathrm{AC}$. In the second step, we demonstrate an improved real-time approach for removing OA online from alpha band of EEG. Furthermore, the application of this approach in the OPTIMI project of the EU's Seventh Framework Programme (FP7) demonstrates the applicability and reliability of our approach.
\end{abstract}

Keywords- EEG; Home Care; Ocular Artifacts; Signal Processing.

\section{INTRODUCTION}

In Telecare and Telehealth projects, mental health is one of the most important aspects especially with respect to the elderly. In these types of system it is necessary to develop realtime, efficient, portable EEG recording and analysis systems. As there are many noises and interferences when subjects record EEG in houses, it's necessary to remove the noises, especially Ocular Artifacts (OA), $50 / 60 \mathrm{~Hz} \mathrm{AC,} \mathrm{DC} \mathrm{adrift} \mathrm{and}$ so on. The aim of the EEG based home care in OPTIMI is to monitor levels of individual mental disorders and collect feedback during treatment using EEG signals. There are 9 online treatment sessions designed to address, for example, Stress, which employs Cognitive Behavioral Therapy (CBT) combined with 'bio-signals' feedback, e.g., EEG.

\section{A. The alpha band of EEG and Importance of alpha rhythm}

The EEG is a record of the oscillations of brain recorded from electrodes on the human scalp. EEG is often labeled according to apparent frequency range: delta $(1-4 \mathrm{~Hz})$, theta (4$8 \mathrm{~Hz})$, alpha $(8-13 \mathrm{~Hz})$, beta $(13-20 \mathrm{~Hz})$, and gamma (roughly $>20 \mathrm{~Hz}$ ). The scalp electric potential amplitude is typically 20 to $100 \mu \mathrm{V}$; its specific shape depends on the subject's state of relaxation [1]. However, some recent studies have suggested that alpha frequencies are an oscillatory component of the human EEG. Furthermore, attention and semantic memory demands can led to a selective suppression of alpha rhythm in different 'subbands', due to visual stimulation as documented in [2].With the eyes open, eye movements cause changes to the electric fields around the eyes and consequently over the scalp, leading to the alpha band of EEG being contaminated by OA. Research has shown that in visual stimulation experiments, alpha extraction with eyes open is necessary and of considerable importance.

\section{B. Removal of ocular artifacts in alpha band}

Considering of the importance of alpha rhythm in many application fields, such as mental illness, individual identification, E-learning, etc, it is necessary to design a procedure to decrease artifacts from the alpha band in EEG recording. Additionally $\mathrm{OA}^{\prime}$ frequency band $(0-16 \mathrm{~Hz})$ is observed to overlap with EEG signals. Meanwhile, single channel recording is applied to subjects to provide a convenient, quick, efficient EEG recording environment and achieve the ubiquitous computing and reduce the intrusion of numerous electrodes. It is noted that because of the strong overlapping background ocular artifacts and deployment of mono-polar recording, neither the existing frequency base de-noising techniques nor statistical methods are able to remove ocular artifacts in alpha band. Therefore, effective filtering becomes extremely difficult and important for both automated and visual analysis of EEG signals. The ocular artifacts, a non-cortical activity, produce large electrical potential around the eyes, which increase the difficulty in analyzing and processing the real EEG data, since it is hard to obtain real information of the brain. The problems of ocular artifacts cancellation and alpha feature extraction of EEG signals are widely discussed in the field of EEG research.

\section{Our method implemented in OPTIMI}

To achieve the OPTIMI objectives, we have developed a 
Mobile EEG Belt, which is a wireless EEG collection device integrated with cognitive interface to provide data to an online mental treatment system (shown in figure 1). In contrast with EEG products in the market, the Mobile EEG Belt uses limited poles to address specific mental disorders, and the belt with electrodes can be altered to match the requirements of monitoring a variety of mental disorders. The algorithms implementing de-noise, features extraction and classification have been incorporated into the Mobile EEG Belt. Additionally, it is easier for users to wear the forehead electrodes than to wear them over their hair; however, due to the close proximity to the eyes there is a need for more strictly enforced de-noise requirements. The realization of effective and efficient 'real-time' interactions with the interface is a clear requirement. Therefore, it is necessary to develop or integrate an innovative methodology and algorithm(s) to meet these needs.

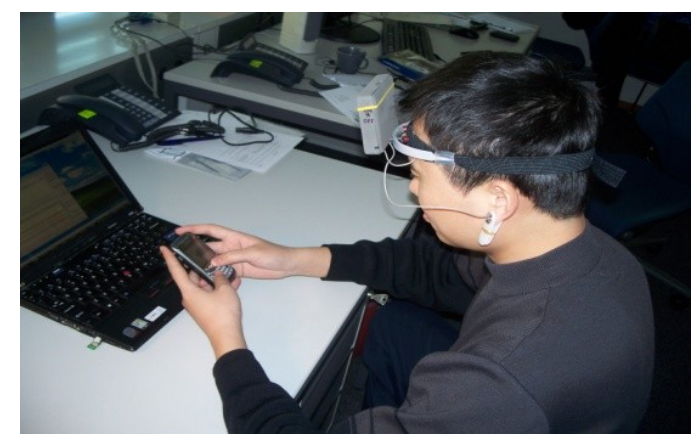

Figure 1. The home care application in OPTIMI project.

\section{The structure of this paper}

The paper is structured as follows: Section 2 provides a brief overview of related works in removing ocular artifacts and feature rhythm extraction algorithms of recent years. Section 3 provides the paper's theoretical background by defining WPT and ICA. Section 4 provides the prior knowledge which will be used in the proposed modified algorithms, and then describes the theoretical foundations have been used in the proposed method and implementation details. The experiments, results, discussion and detailed figures are presented in section 5. Section 6 gives an application of the proposed modified method, and the results show a high precision and effectiveness of the proposed approach. The paper is concluded in section 7 .

\section{RELATED WORKS AND ALGORITHM}

Frequency-based de-noising techniques are the most common methods used in frequency 'subbands' extraction. The Wavelet transform [3] approach is becoming more and more popular for the analysis of biomedical data. A technique has been proposed by [4] for detection and subsequent denoising of these ocular artifacts using Haar wavelets of high orders. However, because this method is based on the high amplitude of the ocular artifacts, if the artifacts' amplitude is not much higher than the normal EEG data, the ocular artifacts will not be detected with precision. WPT is a well-suited technique to analyze special rhythms according to different frequency domain. Wavelets are effective in extracting both time and frequency domain information from time-varying and non-stability EEG signal, extracting alpha rhythms by decomposing the EEG wave into different frequency domains.

However, the major drawback of these Frequency-based techniques is that the ocular artifacts with frequency domain overlapping of alpha rhythms cannot be removed efficiently. We have therefore considered the idea of using ICA to eliminate noise such as ocular artifacts, by using reference signals to characterize ocular artifacts in the known frequency band [5]. ICA is currently a popular method for blind source separation (BSS) of linear mixtures, and is a major tool used to solve nonlinear problems. It aims at recovering independent sources from mixture EEGs, without knowing the mixing matrix and specific knowledge of the sources [6]. Typically, it assumes a model where observations are assumed to be linear mixtures of statistically independent sources. However, ICA can only be used on the condition of multi-channel EEG recording.

This paper proposed to use ICA and WPT for removing the ocular artifacts from the alpha band within a single channel EEG recording. MATLAB and its toolbox FastICA is used to processes EEG data. In the following section, the algorithms used in this paper will be discussed briefly.

\section{IMPROVED ALGORITHMS AND PRIOR KNOWLEDGE}

ICA had been originally proposed to resolve the cocktail party problem which can separate the ocular artifacts from the raw EEG signals with respect to the condition of multichannel. However, the mono-polar recording EEG cannot be processed by traditional ICA. So the ICA will be banded together with WPT to remove ocular artifacts from monopolar recording EEG.

In the mono-polar recording of EEG signal, as is shown in figure 2, the assumption is made that there are many independent signal sources to giving out independent signals, which could be denoted by $\mathrm{S}_{1}(\mathrm{t}), \mathrm{S}_{2}(\mathrm{t}), \mathrm{S}_{3}(\mathrm{t}), \ldots \ldots, \mathrm{S}_{\mathrm{n}}(\mathrm{t}) ; \mathrm{t}$ represents the time index. In order to providing a convenient, pervasive, quick and efficient EEG recording environment to subjects while avoiding hair interference, is obtained by using only one electrode, which is placed on FPz according to the International 10-20 System, as is shown in figure 2. Hence there is only one observed signal recorded, which we could denote by $X(t)$. It can be expressed in the linear equation:

$$
X(t)=a_{1} S_{1}(t)+a_{2} S_{2}(t)+a_{3} S_{3}(t)+\ldots \ldots+a_{n} S_{n}(t)
$$

Where $a_{1}, a_{2}, a_{3}, \ldots . ., a_{n}$ are the parameters that depend on the distance of the recorded electrode from the independent sources. 
Presents the original sources of th EEG signals (components)

Presents the observed signals which we can get

Figure 2. It shows that the relationship between observed signal $\mathrm{X}(\mathrm{t})$ and the independent sources $\operatorname{Si}(\mathrm{t})$.

In this paper, it is important to extract the only the desired components: The ocular artifacts and the alpha band. In order to remove ocular artifacts, there is only one recorded signal X (t) needed. Ideally, any time delays and other factors are omitted from this experiment. Also several assumptions must be made: The first one is that the two independent sources are not completely overlapping in frequency domain, the signals are completely independent under ideal conditions, and basic FIR filter can be used to separate the two frequency independent signals. If the independent sources are not completely overlapping in the frequency domain, the reference signals, which have high correlation with ocular artifacts and low correlation with EEG signals, could be estimated by WPT. Furthermore, OA and EEG signals are independent since eye movements and blinks have different generator mechanisms and produce different field patterns at the scalp [7].

From this prior knowledge, we can see that alpha rhythm frequency band is $8-13 \mathrm{~Hz}$, and ocular artifacts are $0-16 \mathrm{~Hz}$. The main power of ocular artifacts are concentrated at around $5 \mathrm{~Hz}$, the ocular artifacts reference signals can be constructed with signals within the range of $0-7.5 \mathrm{~Hz}$. WPT can be used to extract these reference signals as the input of FastICA, and then the ocular artifacts in the alpha band may be removed. As is mentioned above, in this paper, we introduce a new algorithm which is suitable for the removal of ocular artifacts from their linear mixtures. WPT and ICA are used for the ocular artifacts cancellation in the alpha band.

\section{IMPROVED APPROACH OF OCULAR ARTIFACTS REMOVE FROM THE ALPHA RHYTHM IN EEG}

Because EEG recordings are contaminated by ocular artifacts, the precision of frequency measurements with respect to EEG signals is poor with low resolution and serious interference. We could regard them as the independent components since ocular artifacts have different generator mechanisms and produce different field patterns at the scalp [7]. Based on this, ICA can be used to eliminate the ocular artifacts with frequencies overlapping that of the alpha wave.

The model of ICA requires Multi-channel signals. However, there is only one single channel data, so reference signals are required. Here we have proposed a novel way to obtain reference signals by using WPT. Daubechies 7 (up to 7 levels) was applied as the basis function to decompose the contaminated EEG in order to obtain reference signals. Since the frequency domain of ocular artifacts is $0-16 \mathrm{~Hz}$ and the alpha rhythm frequency band is $8-13 \mathrm{~Hz}$, in order to remove ocular artifacts and reconstruct the ocular artifacts roughly, the 'estimated' decomposition nodes were selected in the frequency band $0-7.5 \mathrm{~Hz}$.

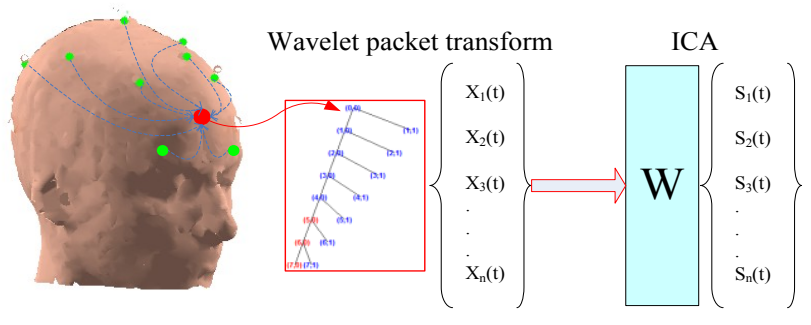

Figure 3. The alpha extraction model based on WPT and ICA.

The ICA model requires the independent sources of the signals $M$ and the observed signals $N$ should satisfy the condition of $\mathrm{M}<=\mathrm{N}$. In this experiment, $\mathrm{M}=2<\mathrm{N}=4$, it meets the requirement of ICA.

The alpha extraction procedures used in this paper (shown in figure 3 ) are briefly given below:

1. Decompose raw data: Apply WPT Daubechies 7 (up to 7 levels) to decompose the contaminated EEG, and selects specific nodes which frequency bands between $0-7.5 \mathrm{~Hz}$, apply inverse WPT to selected wavelet packet coefficients to reconstruct the nodes as the ocular artifacts reference signals. In this paper, last three nodes are selected (make sure the reference signals' frequency domain does not overlap with those of the Alpha wave (or just a little)).

2. Construct the input mixed matrix and using FastICA: Take these ocular artifacts reference signals and raw EEG signal as the input of the ICA mixing matrix, fixed point algorithm with a deflation approach is used to remove ocular artifacts from the alpha band.

3. Independent Components (ICs) selection: Calculate and compare the correlation between the input signals and output signals, Select the independent component which has the largest correlation coefficients with raw EEG signal, $\mathrm{IC}_{\text {EEG_alpha, }}$ to obtain the EEG without the ocular artifacts.

4. Obtain the alpha wave using WPT: Apply WPT to decompose the de-noised EEG, Select specific nodes, and apply inverse WPT to wavelet packet coefficients to reconstruct the alpha wave.

\section{EXPERIMENT AND EVALUATION}

In order to prove our algorithm's effectiveness, it will be compared with wavelet transform method proposed by [8]. In this experiment, the mobile EEG belt (shown in figure 4), which is a wireless EEG collection device integrated with cognitive interface to provide data to an online mental treatment system, is used to collect EEG data. The procedure starts by acquiring EEG data following standard guidelines from healthy college students. The ground reference cable is placed on of the left ear (A1). Each subject sits in a chair with 
eyes closed quietly for a period of 1 minute, and then continuous EEG signals are recorded for two minutes with eyes open and eye-blinks occurring frequently. Since each electrode is chosen to be the input of the algorithm, the ocular artifacts are extremely high when the single electrode is placed near the eyes. Based on the reasoning above, the signals are digitized at a rate of $260.4 \mathrm{~Hz}$ and need to be further filtered.

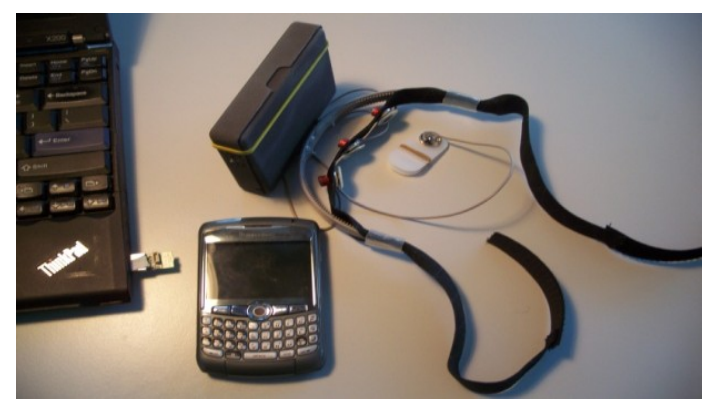

Figure 4. Mobile EEG Belt.

\section{A. Experiment}

The raw EEG data will be pre-processed to remove DC adrift and $50 / 60 \mathrm{~Hz} \mathrm{AC}$ by using hanning window filter. The pass band is from 2 to $40 \mathrm{~Hz}$. Raw EEG signals (marked as (1)) and filtered signals (marked as (2)) are shown in figure 5 .
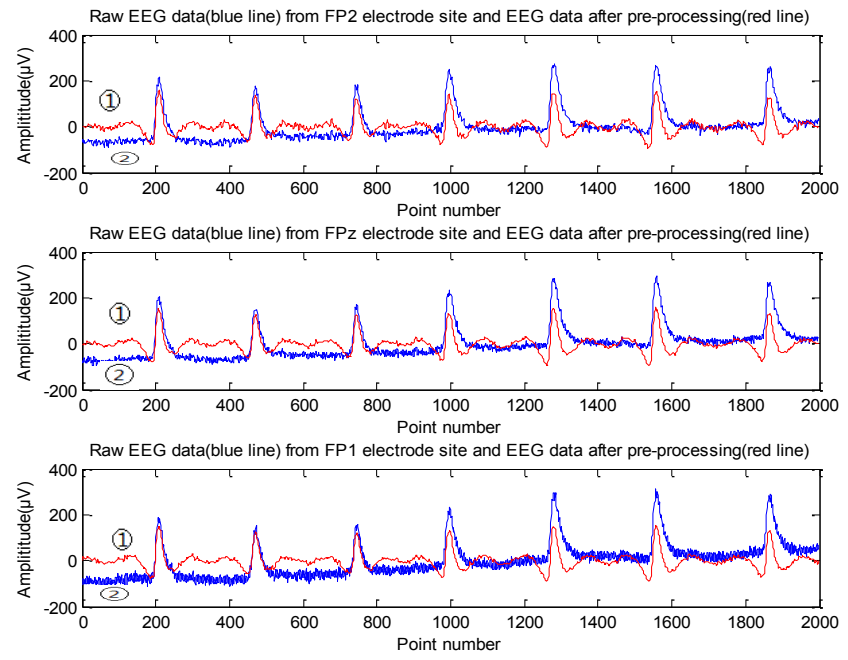

Figure 5. The raw EEG data will be pre-processed to remove DC adrift and $50 / 60 \mathrm{~Hz} \mathrm{AC}$ by using hanning window filter.

The amplitude of alpha wave is changed because of the limitation of ICA own; it can be corrected by the prior knowledge and the ratio as

$\mathrm{R}=$ Raw_alpha_sum/final_alpha_sum;

Where

Raw_alpha_sum= the summation of peak-to-peak value of the alpha wave which is extracted by WPT.

final_alpha_sum= the summation of peak-to-peak value of the alpha wave which is extracted by modified WPT and ICA.
$\mathrm{R}$ is used to multiply by the alpha wave which is extracted by modified WPT and ICA to obtain the normal amplitude alpha wave.

Lastly, WPT is used to extract the alpha wave from the de-noised EEG signal in traditional way, Daubechies 7 (up to 7 levels) is applied as the basis function to decompose the denoised EEG data, and then a calculation of the minimum nodes should be used to reconstruct the alpha rhythm.

\section{B. Results and Discussion}

Results in figure 6 indicate that our method has greater accuracy and efficiency than the WT method. The WT method uses non-adaptive hard threshold function $\left(1.5^{*}\right.$ standard deviation) with window size two seconds. The results indicate that the ocular artifacts are removed, but it can be see that this method retains the background EEG, and spikes are introduced near the artifacts zone, and, most importantly, since this method is based on the high amplitude of the ocular artifacts, if the artifacts' amplitude is not much higher than the normal EEG data, the ocular artifacts will not be detected very well.

According to the time domain plots of raw EEG, selected from two samples, as is shown in figure 6 ( $a$ and $b$ ), it is clear that our method maintains the pure EEG signals while removing ocular artifacts compared with the WT method. Moreover, no spikes are introduced and the ocular artifacts amplitude is greatly reduced. It can also be seen from the average power spectrum in alpha band $(8-13 \mathrm{~Hz})$ of 20 samples in figure 6(c), that when our method is applied to the alpha extraction, the elimination of ocular artifacts is better than WT method. Furthermore, the modified method enhances the signal noise ratio, much better than that extracted by WT method.
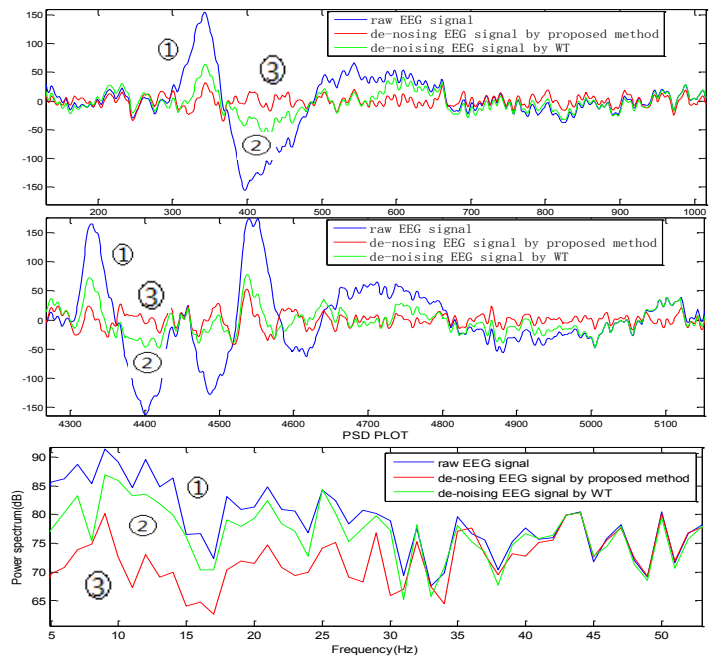

Figure 6. (a), (b) time domain plot of two strips of EEG: the time domain plot of raw EEG signals (blue line, marked as (1)), de-noised EEG by WT (green line, marked as (2)) and de-noised EEG by proposed method (red line, marked as (3) ). (c)the average power spectral density plot: raw EEG signals (blue line, marked as (1)), de-noised EEG by WT method (green line, marked as (2)) and de-noised EEG by proposed method (red line, marked as (3)). 


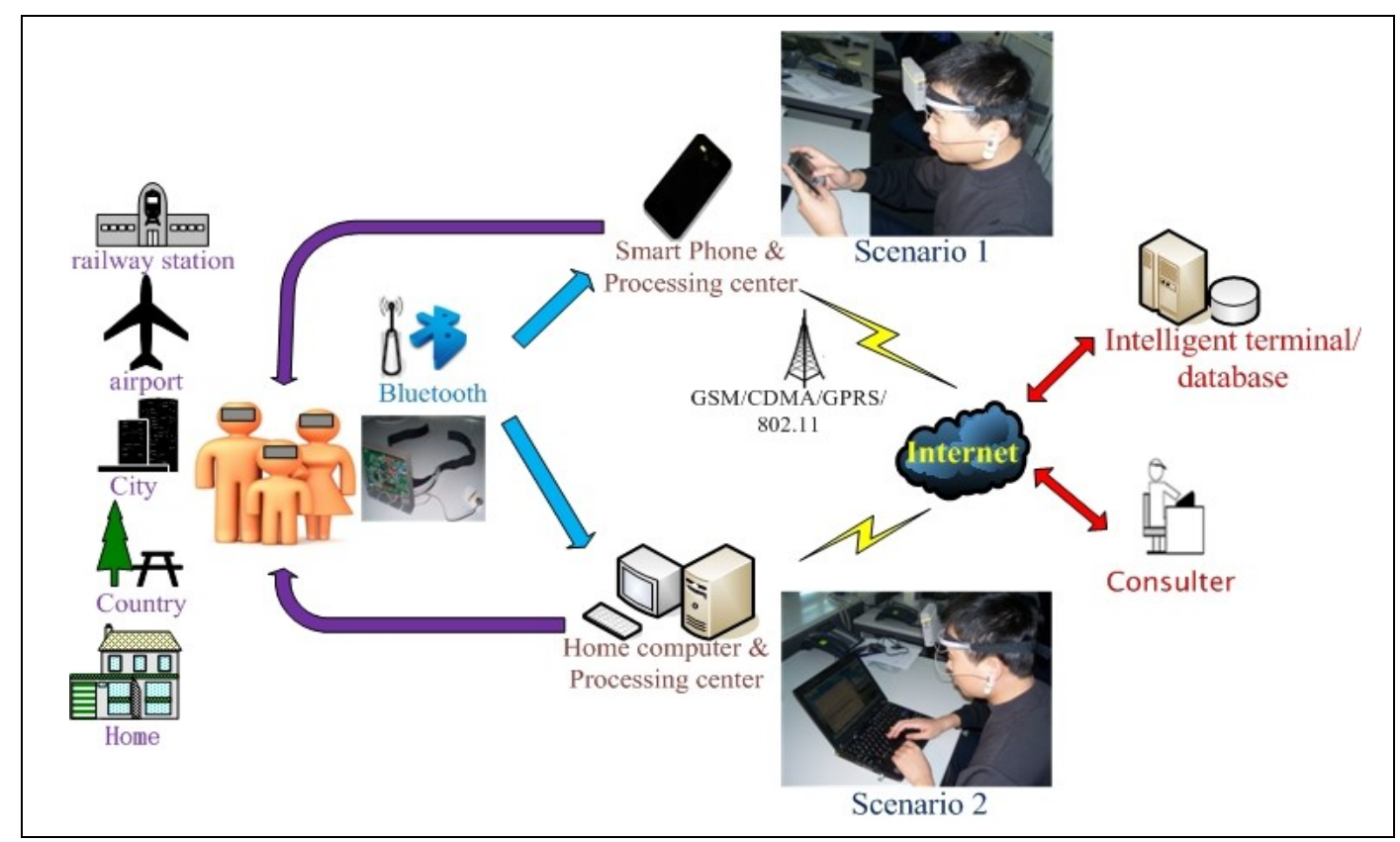

Figure 7. The application of OPTIMI: Home care (while the signal processing algorithms are in the Processing center).

These results also show that our method combines the advantages of WPT in frequency domain analysis and ICA in time domain analysis, as well as overcoming the shortcomings of ICA: it cannot be used in single channel EEG de-noising and WPT where the removal of ocular artifacts from EEG alpha rhythm is not possible due to their wrapped frequency domain.

\section{APPLICATION}

In long term home care, depression and stress-related disorders of the elderly are among the most common mental illnesses. The prevention of these illnesses is one of the central focus points in the European Pact for Mental Health and Well Being yet, currently, very little research is devoted to develop effective systems for early Detection and Prevention of the onset of mental illness. Against this background the goal of OPTIMI is to develop tools to perform Prediction through early identification of the onset of such illness, especially those being stress-related, by monitoring mood states, coping behaviour and changes in stress-related physiological variables (e.g. EEG, heart rate, etc.). In order to identify the precedents and onset of a mental illness, OPTIMI will first identify high stress, especially in high-risk individuals.

In our application as is shown in figure 7, the Mobile EEG Belt is allowed to be used in lots of locations, such as airport, at home, even in the country. When EEG signals are recorded by our device, the EEG signals will be sent to the mobile phone or your own computer, which has the software in it, by Bluetooth. The signal processing model will process the EEG signals, such as de-noising, feature extraction and classifier. Moreover the detection of your level of depression and stress-related disorders will be detected and send to the intelligence terminal or database, and the doctor will monitor your state.

The spontaneous or continuous EEG reflects brain activity measured from surface electrodes and consists of several rhythms originating from known subsystems of the brain, plus event-related components and irregular activity. There have been several attempts at linking depressive mood to spontaneous EEG properties. The majority of studies targeting depression reported on characteristic differences in alpha activity recorded from electrodes placed on frontal locations. For instance, frontal asymmetry quantifies the EEG differences between the hemispheres based on a concept of mood and function of the hemispheres.

\section{CONCLUSION}

In today's society, there is a strong need for technologies that can help to deliver social and health care in the home. These technologies, based around OPTIMI, may be used to inform the processes of long term care. However these technologies all require pure EEG signals in order to assess the states of the subjects under observation. In this paper, based on an OPTIMI application, a modified method for signal processing is proposed. Results indicated that the modified method does not affect the alpha components in the raw EEG zones and ocular artifacts are removed efficiently, the background EEG signals are retained, no spikes are introduced, and the ocular artifacts amplitude is greatly reduced. In addition, the ocular artifacts in EEG signals are considerably reduced using the proposed method irrespective of the amplitude. This hybrid method can reduce the impact of ocular artifacts which overlap in the frequency domain in alpha bands and improve the signal noise ratio compared to the wavelet 
transform method. Moreover, it overcomes the shortcomings of ICA which cannot be used in single EEG recording signal de-noising and WPT which cannot extract alpha waves accurately when ocular artifacts overlap in the frequency domain. Finally, the results of a practical application for EEG ocular artifacts elimination has been demonstrated, which verifies the validity and effectiveness of the proposed approaches.

In the future, we will be engaged in designing reference signals to extract feature signals and other methods for highly artifact selective detection and de-noising in order to obtain feature waves. Although the performance of EEG artifacts processing is validated by visual inspection of experimental data, it is noted that artifact removal never produces perfect results. This imperfection is difficult to evaluate as it is generally not clear what a perfect EEG waveform should look like. Development of criteria to assess artifact removal will become a pressing topic in the future.

\section{ACKNOWLEDGMENT}

This work was supported by National Natural Science Foundation of China (grant no. 60973138), the EU's Seventh Framework Programme OPTIMI (grant no. 248544), the Fundamental Research Funds for the Central Universities (grant no. lzujbky-2009-62), the Interdisciplinary Innovation Research Fund for Young Scholars of Lanzhou University (grant no. LZUJC200910) and the Fundamental Research Funds for the Central Universities (grant no. lzujbky-2010-175).

\section{REFERENCES}

[1] PAUL L.NUNEZ RAMESH SRINIVASAN, (2006). Electric fields of the brain. The Neurophysics of EEG.

[2] Wolfgang Klimesch, (1999). EEG alpha and theta oscillations reflect cognitive and memory performance: a review and analysis, Brain Research Reviews, 29, pp.169-195.

[3] R.J. Croft, R. J.Barry, Neurophysiol Clin, (2000). Removal of ocular artifact from the EEG: a review, 30, 5-19. 10.

[4] S. Venkataramanan, N.V. Kalpakam, J.S. Sahambi (2004), A Novel Wavelet Based Technique for Detection and De-Noising of Ocular Artifact in Normal and Epileptic Electroencephalogram, Proceedings of the 6th Nordic Signal Processing Symposium - NORSIG 2004.

[5] Barros AK, Vigario R,et al. Extraction of event-related signals from multichannel bioelectrical measurements [J]. IEEE Transaction on Biomedical Engineering, 2000, 47(5): pp.583-588.

[6] Ricardo Nuno Vig irio, (1997). Extraction of ocular artefacts from EEG using independent component analysis, Electroencephalography and clinical Neurophysiology, 103, pp.395-404.

[7] Otavio G. Lins, Terence W. Picton, Patrick Berg, and Michael Scherg, (1993). Ocular Artifacts in EEG and Event-Related Potentials I: Scalp Topography. Brain Topography, Volume 6, Number 1.

[8] Mrs V Krishnaveni, Dr S Jayaraman, Mr N Malmurugan, $\operatorname{Dr} \mathrm{A}$ Kandaswamy, Dr K Ramadoss, (2004), Non adaptive thresholding methods for correcting ocular artifacts in EEG, Academic Open Internet Journal, Volume 13. 